\title{
Rapid and reliable diagnostic method to detect Zika virus by real-time fluorescence reverse transcription loop-mediated isothermal amplification
}

\author{
Xu-Guang Guo ${ }^{1,2+}$, Yong-Zhuo Zhou ${ }^{3 \dagger}$, Qin $\mathrm{Li}^{4 \dagger}$, Wei Wang ${ }^{5,6}$, Jin-Zhou Wen ${ }^{7}$, Lei Zheng ${ }^{1}$ and Qian Wang ${ }^{1 *}$
}

\begin{abstract}
To detect Zika virus more rapidly and accurately, we developed a novel method that utilized a real-time fluorescence reverse transcription loop-mediated isothermal amplification (LAMP) technique. The NS5 gene was amplified by a set of six specific primers that recognized six distinct sequences. The amplification process, including 60 min of thermostatic reaction with Bst DNA polymerase following real-time fluorescence reverse transcriptase using genomic Zika virus standard strain (MR766), was conducted through fluorescent signaling. Among the six pairs of primers that we designate here, NS5 was the most efficient with a high sensitivity of up to $3.3 \mathrm{ng} / \mu \mathrm{l}$ and reproducible specificity on eight pathogen samples that were used as negative controls. The real-time fluorescence reverse transcription LAMP detection process can be completed within $35 \mathrm{~min}$. Our study demonstrated that real-time fluorescence reverse transcription LAMP could be highly beneficial and convenient clinical application to detect Zika virus due to its high specificity and stability.
\end{abstract}

Keywords: Zika virus, NS5 gene, LAMP, Loop-mediated isothermal amplification, Real-time fluorescence reverse transcription

\section{Introduction}

Zika virus is primarily transmitted by the bite of the female Aedes aegypti mosquito in tropical regions, which must feed on blood to lay eggs (Gulland 2016; Walsh 2016). Other species of mosquito have been reported such as Aedes polynesiensis and Aedes albopictus. The virus has also been isolated from a number of arboreal mosquito species in the Aedes genus.

Patients with active or recessive infection and nonhuman primates infected with Zika virus are the source of the disease. The symptoms of infection are similar to dengue fever and include fever, rash, joint pain, muscle pain, headache and conjunctivitis (Interlandi 2016).

\footnotetext{
${ }^{*}$ Correspondence: wangqian@smu.edu.cn

${ }^{\dagger}$ Xu-Guang Guo, Yong-Zhuo Zhou and Qin Li contributed equally to this work

${ }^{1}$ Laboratory Medicine Center, Nanfang Hospital, Southern Medical University, Guangzhou 510515, Guangdong Province, China

Full list of author information is available at the end of the article
}

Only $20 \%$ of infected people present clinical symptoms, including fever, rash, joint pain and conjunctivitis. The symptoms usually disappear in less than a week. However, Zika virus could pose serious problems for pregnant women and their fetus. Zika can be transmitted to the fetus in the uterus or during the delivery process (Chen and Tang 2016). Zika virus nucleic acid has been detected in milk. In early 2015, a widespread epidemic of Zika fever, caused by the Zika virus in Brazil, spread to other parts of South and North America. The incidence in China is relatively low but the number is steadily increasing. Zika virus infection has become a serious global public health threat to humans (Worobey 2017). The outbreak period is usually associated with the mosquito breeding season. Thus, a fast and efficient method to detect $\mathrm{Zika}$ virus is necessary.

Real-time loop mediated isothermal amplification (LAMP) is a novel technology that has been developed in recent years (Xia et al. 2014). LAMP is a thermos-stable 
nucleic acid amplification technique for gene detection that requires 4-6 primers targeting 4-6 specific sequences (Mansour et al. 2015). It only takes $60 \mathrm{~min}$ for the Bst DNA polymerase to amplify the target sequences. LAMP technology has been widely used in detecting a variety of viruses, bacteria and parasites, which has been useful for pathogen identification in clinics (Khan et al. 2012).

Compared with traditional serologic test, LAMP is fast and efficient and may be a particularly useful method for infectious disease diagnosis in low and middle-income countries. LAMP does not require expensive equipment, and the process can be performed with a water bath or incubator (Hattori et al. 2016). Thus far, detection methods for Zika virus have primarily relied on traditional real-time polymerase chain reaction (RT-PCR). In this study, we demonstrated that Zika virus can be accurately detected by LAMP and that it is less time-consuming and has a higher sensitivity than traditional RT-PCR, suggesting that this method could be beneficial tothe clinical treatment and prevention of Zika infections.

\section{Materials and methods}

Zika virus and bacterial strains

The nucleic acid of Zika virus MR766 RNA was purchased from Beijing Ruilimaidi Laboratory Co., Ltd. Klebsiella pneumoniae ATCC700603, Acinetobacter baumannii ATCC19606, Streptococcus pneumoniae ATCC49619, Staphylococcus aureus ATCC25923, Streptococcus mitis ATCC49456, Pseudomonas aeruginosa ATCC27853, Haemophilus influenzae ATCC49766 and Escherichia coli ATCC25922 were stored in the microbiology room of the Third Affiliated Hospital of Guangzhou Medical University.

\section{Primer design and synthesis}

The primers forward outer primer (F3), backward outer primer (B3), forward internal primer (FIP), backward internal primer (BIP) and loop backward primer (LB) were designed by Guangzhou Deaou Biotechnology Company Limited and synthesized by Tiangen Biochemical Technology (Beijing) Company Limited. The sequences of the primers used in this study are listed in Tables 1, 2, 3 and 4.

\section{LAMP reaction system preparation}

Bacterial genomic DNA extraction kit was purchased from Tiangen Biochemical Technology Company Limited. The LAMP reaction system was prepared according to the manual for the LAMP amplification kit.
Table 1 The primer NS5-1 used for detection of Zika virus

\begin{tabular}{lll}
\hline Primers & Sequence $\left(\mathbf{5}^{\prime} \rightarrow \mathbf{3}^{\prime}\right)$ & Length $(\mathbf{b p})$ \\
\hline F3 & GCTATGACTGACACCACAC & 19 \\
B3 & ACCTTGGATCATTCACAGC & 19 \\
FIP (F1C+F2) & CCAGCTCCTTCCACAGCCCAAGAAGGTA & 36 \\
& CTCGCCAG & \\
BIP (B1C+B2) & TAAGCGGCCACGTGTCTGAATATTGCTCCCA & 37 \\
& GTGCTG & 20 \\
LoopF & AGGAAGCGACCATGTTCATT & 22 \\
LoopB & GAAGAGTTCATCAACAAGGTGC \\
\hline
\end{tabular}

Table 2 The primer NS5-2 used for detection of Zika virus

\begin{tabular}{lll}
\hline Primers & Sequence $\left(\mathbf{5}^{\prime} \boldsymbol{\rightarrow} \mathbf{3}^{\prime}\right)$ & Length $(\mathbf{b p})$ \\
\hline F3 & CATCTTCCCTCGTGAATGG & 19 \\
B3 & ACCTTGTTGATGAACTCTTCTT & 22 \\
FIP $($ F1C + F2) & CGGTGTGGTGCAGTCATAGCGGTTGTTAG & 42 \\
& ACTCCTGTCAAA & \\
BIP (B1C+B2) & GACACCAGGGTGCCAGACGGAAGCGACC & 36 \\
& ATGTTCAT & 20 \\
LoopF & CCTGTAACTCCAGTCACCAC & 18 \\
LoopB & AGAAGGTACTCGCCAGGT & \\
\hline
\end{tabular}

Table 3 The primer NS5-3 used for detection of Zika virus

\begin{tabular}{lll}
\hline Primers & Sequence $\left(\mathbf{5}^{\prime} \boldsymbol{\rightarrow} \mathbf{3}^{\prime}\right)$ & Length $(\mathbf{b p})$ \\
\hline F3 & GCCAACAAAGAGTCTTCAAAG & 21 \\
B3 & ACAACTATGGCACTCTCCT & 19 \\
FIP (F1C+F2) & AGACACGTGGCCGCTTACGCCAGGTAATGA & 37 \\
& ACATGGT & \\
BIP (B1C+B2) & GCAGCACTGGGAGCAATATTTGACCTTGGA & 41 \\
& TCATTCACAGC & 18 \\
LoopF & CCTTCCACAGCCAGGAAG & 18 \\
LoopB & AATGGAAGACGGCTGTGG \\
\hline
\end{tabular}

Table 4 The primer NS5-4 used for detection of Zika virus

\begin{tabular}{lll}
\hline Primers & Sequence $\left(\mathbf{5}^{\prime} \boldsymbol{\rightarrow} \mathbf{3}^{\prime}\right)$ & Length $(\mathbf{b p})$ \\
\hline F3 & TCTTCCCTCGTGAATGGG & 18 \\
B3 & ACCTTGTTGATGAACTCTTCTT & 22 \\
FIP $($ F1C + F2) & CGGTGTGGTGCAGTCATAGCTTGTTAGAC & 42 \\
& TCCTGTCAAAGC & \\
BIP (B1C+B2) & GACACCAGGGTGCAGACGGAAGCGACC & 36 \\
& ATGTTCAT & 20 \\
LoopF & CCTGTAACTCCAGTCACCAC & 18 \\
LoopB & AGAAGGTACTCGCCAGGT & \\
\hline
\end{tabular}


Primer screening and dissociation curve analysis

Four sets of primers were designed: NS5-1, NS5-2, NS53 , and NS5-4. Each of the working fluids to Zika virus RNA served as a template. The reaction procedure was set to $63{ }^{\circ} \mathrm{C} 15 \mathrm{~s}, 63{ }^{\circ} \mathrm{C} 45 \mathrm{~s}$ as one cycle for 60 cycles, $95{ }^{\circ} \mathrm{C} 15 \mathrm{~s}$ to terminate the reaction, and $63{ }^{\circ} \mathrm{C} 45 \mathrm{~s}$ at the collection of fluorescence signals. A reaction tube was used for the dissolution curve detection and to compare the amplification efficiency of four sets of primers while screening out the highest amplification efficiency without a primer dimer.

\section{Sensitivity of LAMP for Zika virus}

The RNA concentration of Zika virus was measured by adjusting the RNA template concentration with $1 \mu \mathrm{l}$ of TE buffer to the Thermo Scientific Nanodrop 2000 microphotometer and $1 \mu \mathrm{l}$ of RNA extract of Zika virus. $10 \mu \mathrm{l}$ of RNA stock solution was diluted 10-fold with ultra-pure water four times to obtain five RNA template concentrations. This was added to the LAMP system reaction, and the amplification curve was observed to evaluate the sensitivity of the primers.
Specificity and repeatability of LAMP for Zika virus

The genomic DNA of Zika virus and other negative pathogens were extracted and amplified according to the reaction conditions of Real-LAMP. The specificity of the primers was evaluated. One of the positive strains and five negative strains were tested three times with LAMP. The same primer used for the repeat test was used to evaluate the reliability of LAMP.

\section{Results}

Primer screening test of LAMP assay

Four sets of primers amplified without the loop primer showed that the primers NS5-3 had a peak at about 39 min and that the NS5-1 primers had a peak at about $31 \mathrm{~min}$. The efficiencies are both lower than those with the loop primers. The NS5-2 and NS5-4 primers had no peak (Figs. 1, 2).

At approximately $15 \mathrm{~min}$ after the initiation of the reaction, the system of the primer NS5-3 peaked, and at approximately $21 \mathrm{~min}$, the reaction of the NS5-1 primers peaked. The Bio-Rad CFX Manager detected the amplification signal of the NS5 gene, NS5-2 and NS5-4 without
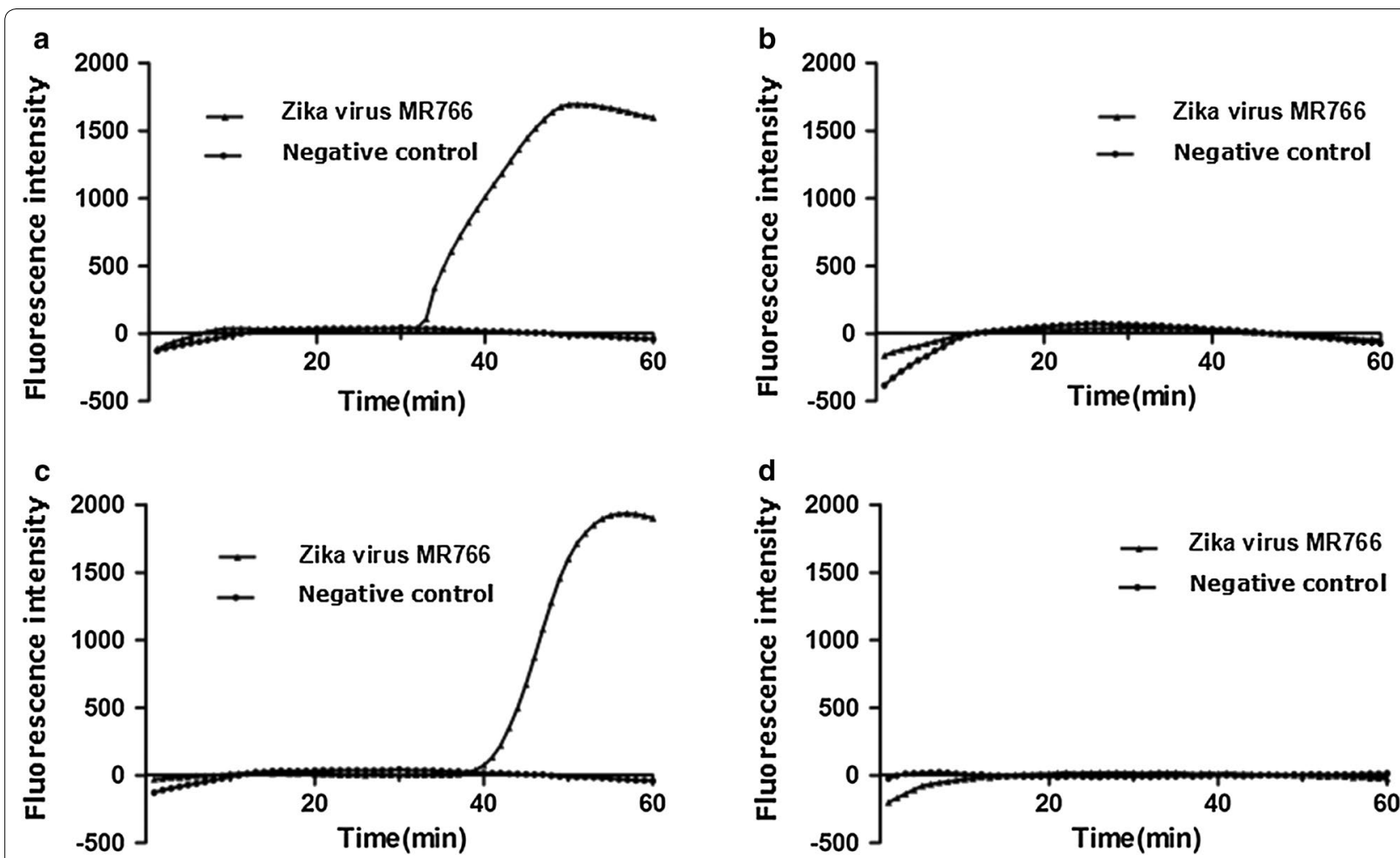

Fig. 1 The primer of the LAMP screening test in this study: a real-amp without loop primers (NS5-1); b real-amp without loop primers (NS5-2); $\mathbf{c}$ real-amp without loop primers (NS5-3); d real-amp without loop primers (NS5-4) 

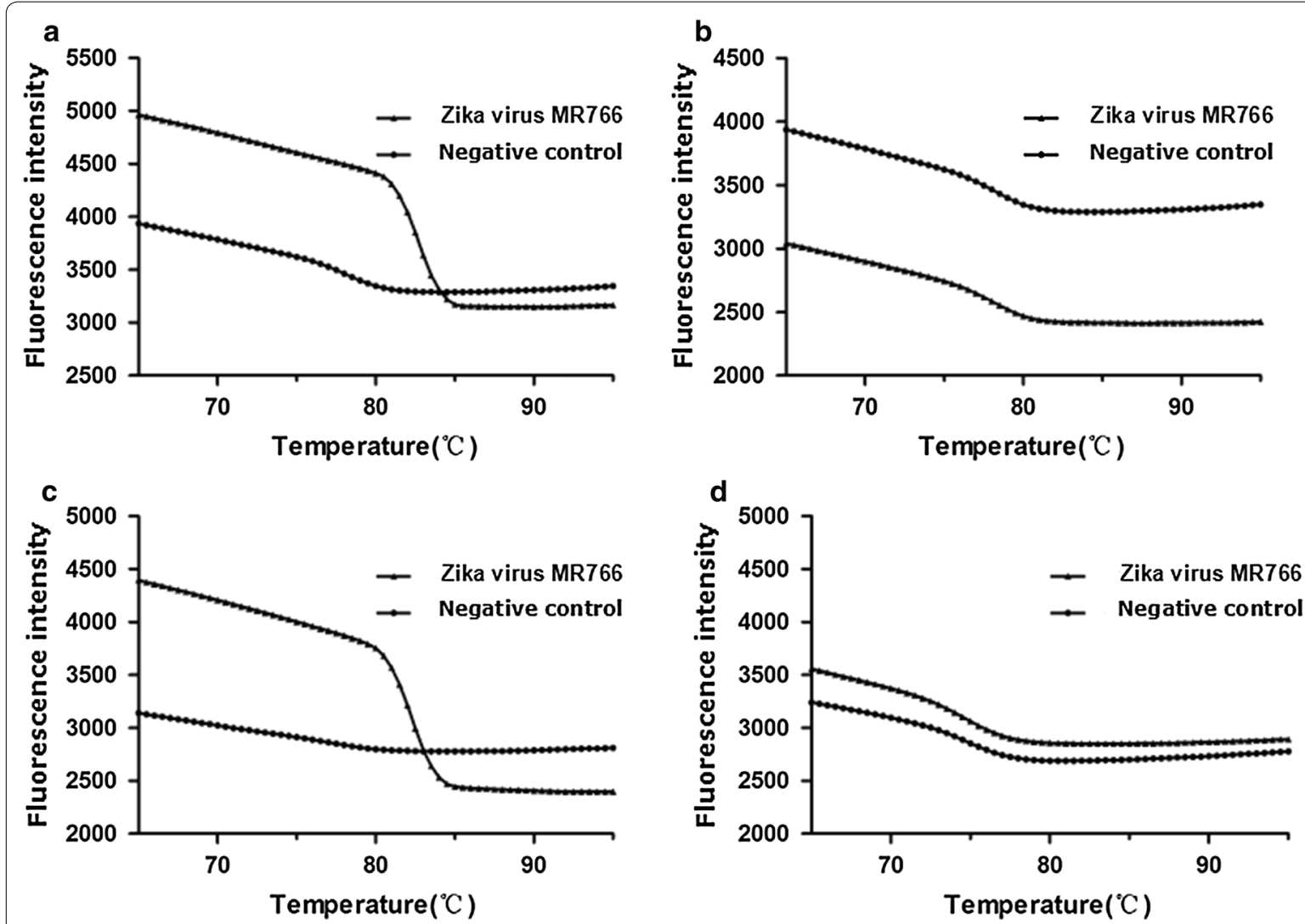

Fig. 2 The LAMP melting curve in this study: a real-amp without loop primers (NS5-1); b real-amp without loop primers (NS5-2); c real-amp without loop primers (NS5-3); d real-amp without loop primers (NS5-4)

a peak (Fig. 3). Although the primer NS5-3 had a relatively high amplification efficiency for the Zika virus NS5 gene, the fluorescence intensity of the negative control curve was significantly decreased, possibly for the dimer of primers (Fig. 4).

\section{Sensitivity of LAMP for amplification of Zika virus}

In this study, the clinical common pathogens were selected as negative controls, but no Zika virus RNA was amplified. The typical "S" curve showed that the specificity of Zika virus RNA was better. The LAMP primer NS5-1 has good specificity and does not cross-react with non-target bacteria (Fig. 5).

\section{Specificity of LAMP for amplification of Zika virus}

Measured by micro-spectrophotometer, the experiment's Zika virus nucleic acid concentration is $33 \mathrm{ng} / \mu \mathrm{l}$. Diluted by 4 gradients, the resulting concentrations were 3.3 , $0.33 \mathrm{ng} / \mu \mathrm{l}, 33$ and $3.3 \mathrm{pg} / \mu \mathrm{l}$. The limit of the detection of this test can reach $3.3 \mathrm{ng} / \mu \mathrm{l}$. Reliable test results are shown in Fig. 6.

\section{Repeatability of LAMP for amplification of Zika virus}

Take Zika virus NS5 for three replicates, wherein two peaks were at $23 \mathrm{~min}$ and another peak was at $20 \mathrm{~min}$. The difference in peak time is approximately $3 \mathrm{~min}$. The repeatability of the tests is shown in Fig. 7.

\section{Discussion}

The Zika virus belongs to the Flaviviridae family and the Flavivirus genus and is thus related to the dengue fever, yellow fever, Japanese encephalitis, and West Nile viruses (Zuanazzi et al. 2017). Most patients show no symptoms or have mild symptoms (Zhang et al. 2017). Only 

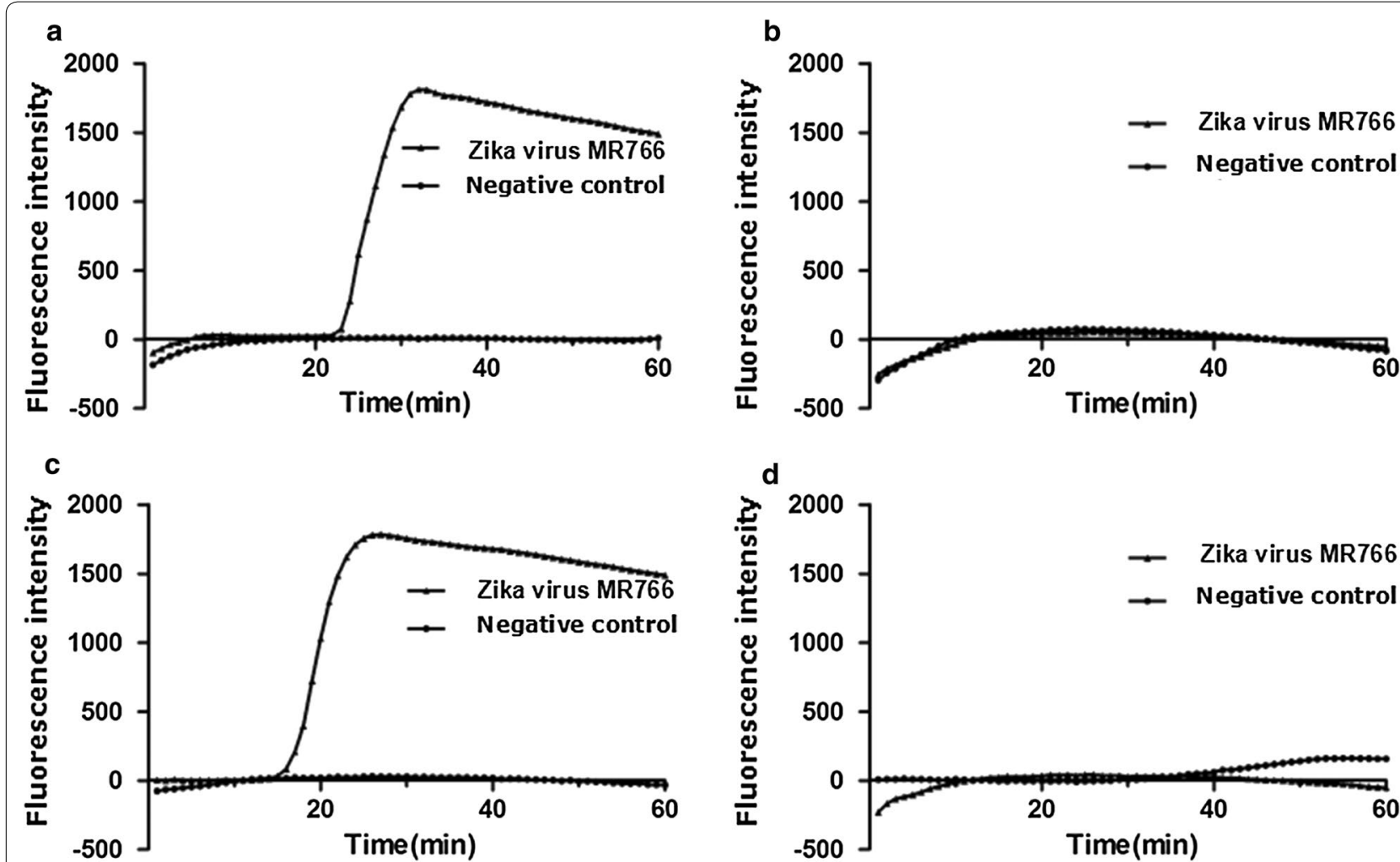

Fig. 3 The primer of LAMP screening test in this study. a Real-amp with loop primers (NS-1); b real-amp with loop primers (NS5-2); c real-amp with loop primers (NS5-3); d real-amp with loop primers (NS5-4)

$20 \%$ of patients experience fever, rash, conjunctivitis and joint pain, while in some cases, symptoms disappear within a week (Wilbe et al. 2017; Zamani and Zamani 2017). Therefore, to develop a rapid and robust nucleic acid amplification assay to efficiently detect Zika virus is critically important for clinical treatment and disease prevention.

In this study, the NS5 gene was selected as the target gene and 8 clinical common pathogens were used as negative controls. The yellow fever virus was theoretically selected as the negative control.

NS5 gene sequences were successfully amplified and displayed as a typical ' $S$ ' shape in Zika virus, except for other negative pathogens, which were used as negative controls, indicating that the NS5 gene sequence as a highly specific candidate could be used in Zika detection. The results showed that the ring primer could significantly shorten the reaction time. The reaction of the ring primer lasted approximately $22 \mathrm{~min}$, and the peak time of the ring primer was less than $10 \mathrm{~min}$. The entire reaction process can be completed within 35 min, which is significantly shorter than that for PCR and is conducive to the clinical diagnosis of Zika virus infection, the timely detection of Zika virus infection, and timely treatment.

Traditional PCR methods are based on agarose gel electrophoresis, which are cumbersome to operate (Wong et al. 2017). Our current method, the LAMP amplification technology, is superior due to its high sensitivity and specificity and ease of operation. Generally, the amplification could be visualized and quantified by fluorescence dye intensity. Primers were specifically labeled with fluorescent dye and embedded in the amplified doublestranded DNA. False positive results could be obtained by the dimer in the primer. In our study, we carefully checked the dissolution curve for the NS5 primer. The negative control curve was straight while the positive control curve was nearly overlapped, indicating that the NS5 primer contained no dimer. 

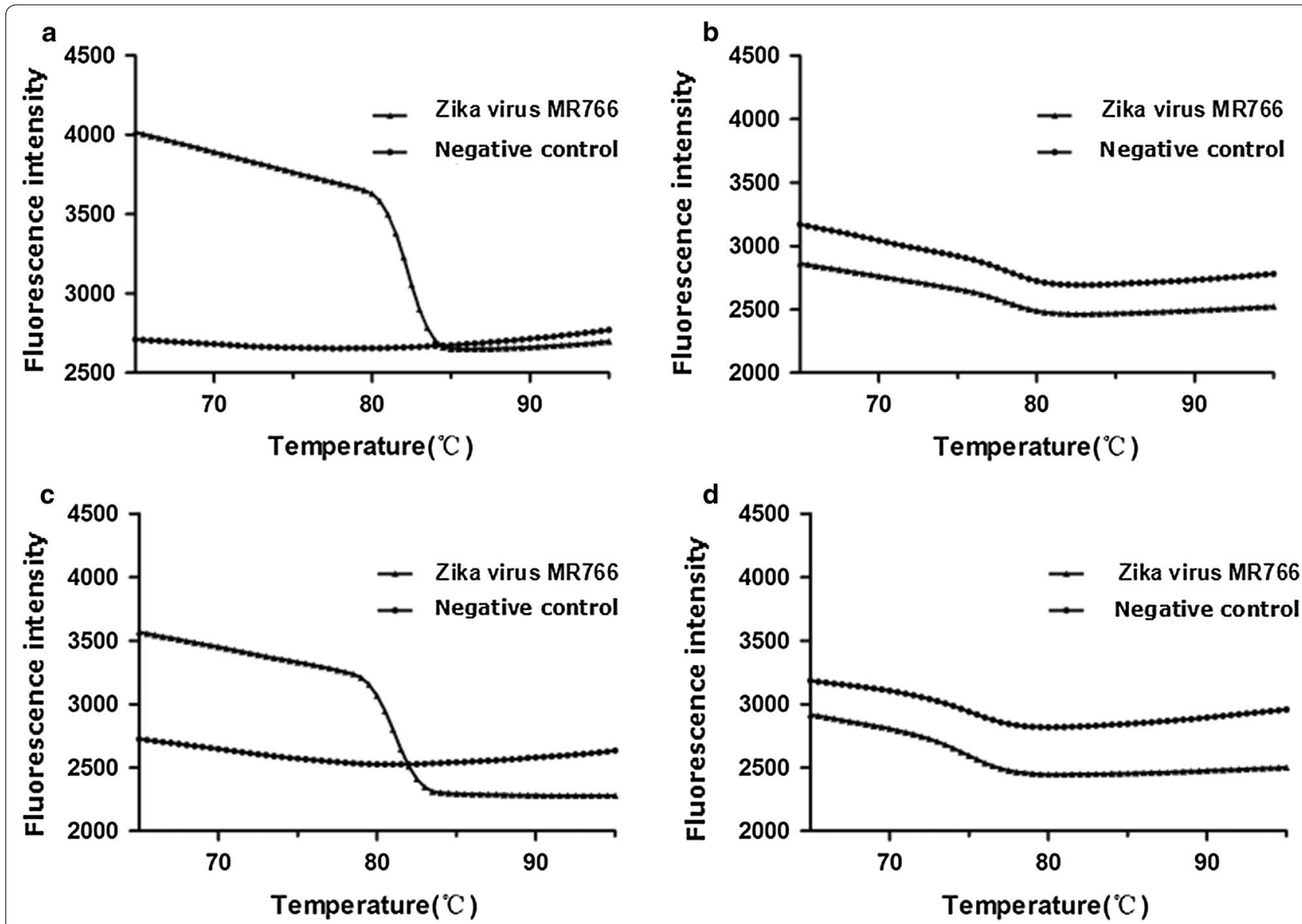

Fig. 4 The LAMP melting curve in this study. a Real-amp with loop primers (NS5-1); b real-amp with loop primers (NS5-2); c real-amp with loop primers (NS5-3); d real-amp with loop primers (NS5-4)

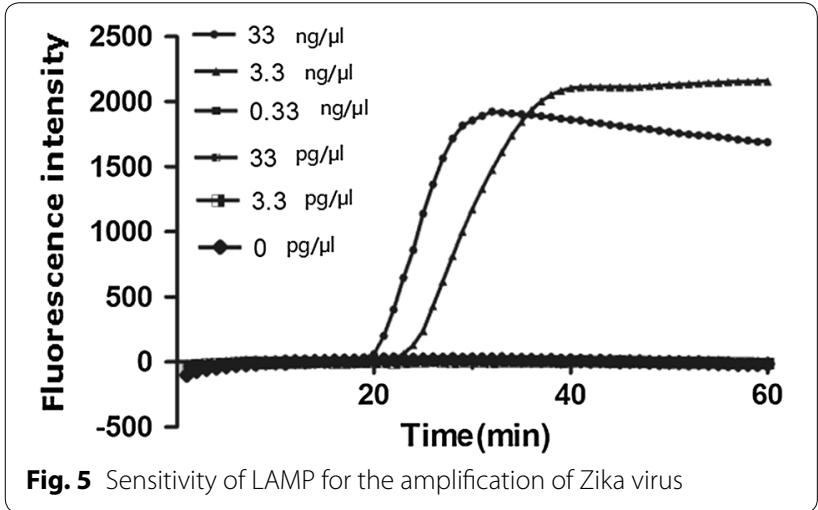

LAMP detection technology has the following advantages compared with other methods. The primers used in the LAMP detection technique are based on six primers designed for the target sequence (Abdul-Ghani et al.
2012; Ahmad and Hashsham 2012; Deborggraeve and Buscher 2012; Ebenezer et al. 2012; Njiru 2012). In addition, LAMP is superior to other methods due to its high sensitivity, as high as $3.3 \mathrm{ng} / \mu \mathrm{l}$. No positive signals were observed in the negative control. These results were reproducible, and we repeated them three times in our study with no significant difference in peak time. An important feature of the LAMP method is the very short detection time and small amount of reagents needed. LAMP is a consistent reaction system that avoids temperature changes, which may influence accuracy.

However, there are limitations. For example, inevitable aerosol pollution may lead to false positive results (Asiello and Baeumner 2011; Fu et al. 2011). LAMP is less common than PCR and is primarily used as a diagnostic or detection technique, not for cloning (Beck and Henrickson 2010). In addition, the LAMP reaction system requires more primers, which may increase the 


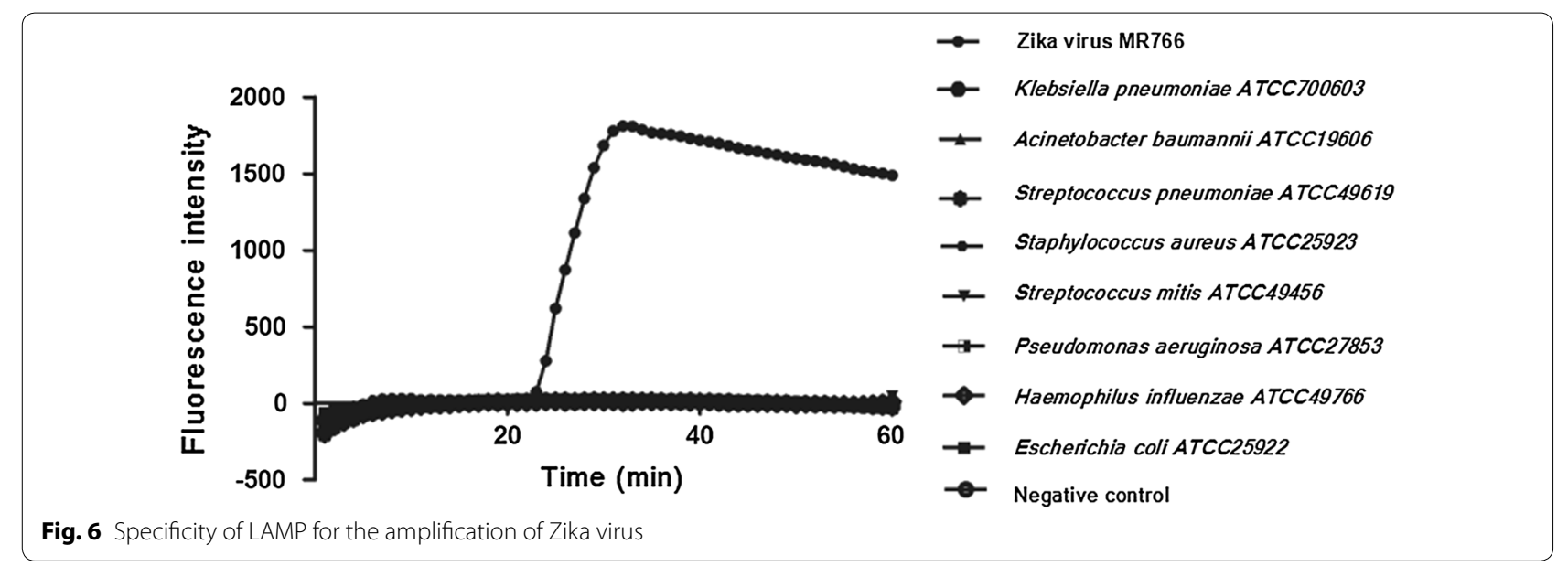

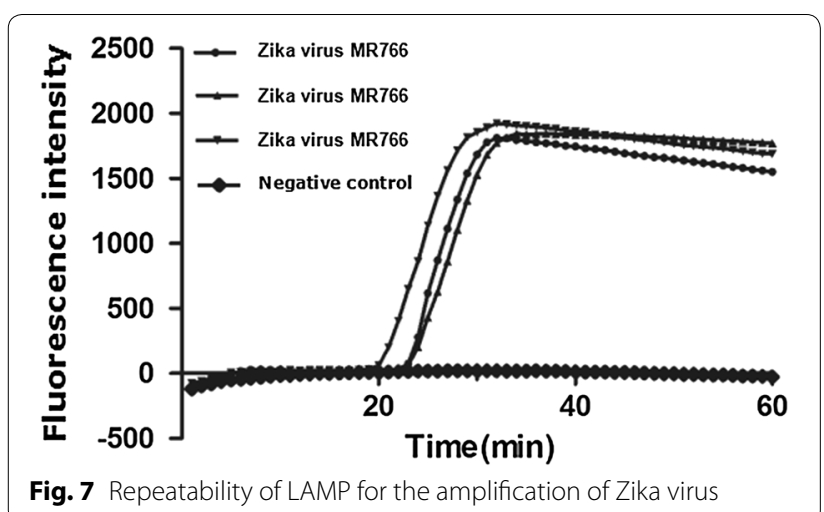

possibility to form dimers (Gill and Ghaemi 2008; Mori and Notomi 2009).

Our current study demonstrated that the application of LAMP to detect Zika virus was superior to traditional methods with a high sensitivity and specificity. As a rapid and efficient novel method, LAMP may be a beneficial tool for clinical application.

\section{Abbreviation \\ LAMP: loop-mediated isothermal amplification.}

\section{Authors' contributions}

XGG and QW designed the study. XGG, QL, WW, YZZ, JZW, LZ and QW collected and analyzed the data. YZZ advised on data analysis. XGG drafted and wrote the manuscript. QL and QW revised the manuscript critically for intellectual content. All authors gave intellectual input to the study. All authors read and approved the final manuscript.

\section{Author details}

${ }^{1}$ Laboratory Medicine Center, Nanfang Hospital, Southern Medical University, Guangzhou 510515, Guangdong Province, China. ${ }^{2}$ Department of Clinical Laboratory Medicine, The Third Affiliated Hospital of Guangzhou Medical University, Guangzhou 510150, Guangdong Province, China. ${ }^{3}$ Department of Clinical Laboratory Medicine, The Third Affiliated Hospital of Southern Medical University, Guangzhou 510150, Guangdong Province, China. ${ }^{4}$ Section of Pulmonary, Critical Care and Sleep Medicine, Department of Medicine, Yale School of Medicine, Yale University, New Haven, CT 06520-8057, USA.

${ }^{5}$ Department of Laboratory Medicine, The First Affiliated Hospital of Shenzhen University, Shenzhen 518035, Guangdong, China. ${ }^{6}$ Department of Laboratory Medicine, Shenzhen Second People's Hospital, Shenzhen 518035, Guangdong, China. ${ }^{7}$ Center For Disease Control and Prevention of Chaozhou, Guangzhou 521000, Guangdong Province, China.

\section{Acknowledgements}

This research was funded by the General Research Projects of Medical and Health Sciences of Guangzhou City (Funding No: 20151A011083), Bureau of Science, Technology and Information of Guangzhou (Funding Nos. 20151217081 and 2016080067).

\section{Competing interests}

The authors declare that they have no competing interests.

\section{Availability of data and materials}

All data generated or analyzed during this study are included within the article.

\section{Consent for publication}

All data published here are under consent for publication.

\section{Ethics approval and consent to participate}

The study was approved by the ethics committees of the participating hospitals and institutes. All procedures performed in the studies involving human participants were in accordance with the ethical standards of the institutional and national research committee and with the 1964 Helsinki declaration and its later amendments or comparable ethical standards.

\section{Funding}

This study was supported by the General Research Projects of Medical and Health Sciences of Guangzhou City (No. 20151A011083), the Bureau of Science, Technology and Information of Liwan District of Guangzhou (Nos. 20151217081 and 2016080067), and the National Nature Science Foundation (No. 81700004)

\section{Publisher's Note}

Springer Nature remains neutral with regard to jurisdictional claims in published maps and institutional affiliations.

Received: 19 February 2018 Accepted: 13 April 2018

Published online: 18 April 2018 


\section{References}

Abdul-Ghani R, Al-Mekhlafi AM, Karanis P (2012) Loop-mediated isothermal amplification (LAMP) for malarial parasites of humans: would it come to clinical reality as a point-of-care test? Acta Trop 122:233-240. https://doi. org/10.1016/j.actatropica.2012.02.004

Ahmad F, Hashsham SA (2012) Miniaturized nucleic acid amplification systems for rapid and point-of-care diagnostics: a review. Anal Chim Acta 733:1-15. https://doi.org/10.1016/j.aca.2012.04.031

Asiello PJ, Baeumner AJ (2011) Miniaturized isothermal nucleic acid amplification, a review. Lab Chip 11:1420-1430. https://doi.org/10.1039/ c0lc00666a

Beck ET, Henrickson KJ (2010) Molecular diagnosis of respiratory viruses. Future Microbiol 5:901-916. https://doi.org/10.2217/fmb.10.48

Chen HL, Tang RB (2016) Why Zika virus infection has become a public health concern? J Chin Med Assoc 79:174-178. https://doi.org/10.1016/j. jcma.2016.03.001

Deborggraeve S, Buscher P (2012) Recent progress in molecular diagnosis of sleeping sickness. Expert Rev Mol Diagn 12:719-730. https://doi. org/10.1586/erm.12.72

Ebenezer V, Medlin LK, Ki JS (2012) Molecular detection, quantification, and diversity evaluation of microalgae. Mar Biotechnol (NY) 14:129-142. https://doi.org/10.1007/s10126-011-9427-y

Fu S, Qu G, Guo S, Ma L, Zhang N, Zhang S, Gao S, Shen Z (2011) Applications of loop-mediated isothermal DNA amplification. Appl Biochem Biotechnol 163:845-850. https://doi.org/10.1007/s12010-010-9088-8

Gill P, Ghaemi A (2008) Nucleic acid isothermal amplification technologies: a review. Nucleosides Nucleotides Nucleic Acids 27:224-243. https://doi. org/10.1080/15257770701845204

Gulland A (2016) WHO's Chan warns over spread of Zika virus beyond Latin America. BMJ 352:i1739. https://doi.org/10.1136/bmj.i1739

Hattori T, Chagan-Yasutan H, Shiratori B, Egawa S, Izumi T, Kubo T, Nakajima C, Suzuki Y, Niki T, Alisjahbana B, Telan E (2016) Development of point-ofcare testing for disaster-related infectious diseases. Tohoku J Exp Med 238:287-293. https://doi.org/10.1620/tjem.238.287

Interlandi J (2016) Will these shirts protect you from Zika? With fears on the rise we put treated clothing to the test. Consum Rep 81:25-27

Khan MG, Bhaskar KR, Salam MA, Akther T, Pluschke G, Mondal D (2012) Diagnostic accuracy of loop-mediated isothermal amplification (LAMP) for detection of Leishmania DNA in buffy coat from visceral leishmaniasis patients. Parasit Vectors 5:280. https://doi.org/10.1186/1756-3305-5-280

Mansour SM, Ali H, Chase CC, Cepica A (2015) Loop-mediated isothermal amplification for diagnosis of 18 World Organization for Animal Health (OIE) notifiable viral diseases of ruminants, swine and poultry. Anim Health Res Rev 16:89-106. https://doi.org/10.1017/s1466252315000018

Mori Y, Notomi T (2009) Loop-mediated isothermal amplification (LAMP): a rapid, accurate, and cost-effective diagnostic method for infectious diseases. J Infect Chemother 15:62-69. https://doi.org/10.1007/ s10156-009-0669-9

Njiru ZK (2012) Loop-mediated isothermal amplification technology: towards point of care diagnostics. PLoS Negl Trop Dis 6:e1572. https://doi. org/10.1371/journal.pntd.0001572

Walsh B (2016) Why the Zika outbreak marks a new normal for infectious disease. Time 187:9-10

Wilbe M, Gudmundsson S, Johansson J, Ameur A, Stattin EL, Anneren G, Malmgren H, Frykholm C, Bondeson ML (2017) A novel approach using long-read sequencing and ddPCR to investigate gonadal mosaicism and estimate recurrence risk in two families with developmental disorders. Prenat Diagn. https://doi.org/10.1002/pd.5156

Wong SJ, Furuya A, Zou J, Xie X, Dupuis AP 2nd, Kramer LD, Shi PY (2017) A multiplex microsphere immunoassay for Zika virus diagnosis. EBioMedicine 16:136-140. https://doi.org/10.1016/j.ebiom.2017.01.008

Worobey M (2017) Epidemiology: molecular mapping of Zika spread. Nature 546:355-357. https://doi.org/10.1038/nature22495

Xia Y, Guo X-G, Zhou S (2014) Rapid detection of Streptococcus pneumoniae by real-time fluorescence loop-mediated isothermal amplification. J Thorac Dis 6:1193-1199. https://doi.org/10.3978/j.issn.2072-1439.2014.07.29

Zamani M, Zamani V (2017) Sexual transmission of Zika virus: an assessment of the evidence. Iran J Public Health 46:1305-1306

Zhang B, Pinsky BA, Ananta JS, Zhao S, Arulkumar S, Wan H, Sahoo MK, Abeynayake J, Waggoner JJ, Hopes C, Tang M, Dai H (2017) Diagnosis of Zika virus infection on a nanotechnology platform. Nat Med 23:548-550. https://doi.org/10.1038/nm.4302

Zuanazzi D, Arts EJ, Jorge PK, Mulyar Y, Gibson R, Xiao Y, Bringel Dos Santos M, Machado M, Siqueira WL (2017) Postnatal identification of Zika virus peptides from saliva. J Dent Res 96:1078-1084. https://doi. org/10.1177/0022034517723325

\section{Submit your manuscript to a SpringerOpen ${ }^{\circ}$ journal and benefit from:}

- Convenient online submission

- Rigorous peer review

- Open access: articles freely available online

- High visibility within the field

Retaining the copyright to your article

Submit your next manuscript at $\boldsymbol{~ s p r i n g e r o p e n . c o m ~}$ 